\title{
Changes in elastic moduli as evidence for quadrupolar ordering in the rare-earth frustrated magnet $\mathrm{Tb}_{2} \mathrm{Ti}_{2} \mathrm{O}_{7}$
}

\author{
Y. Gritsenko $\odot,{ }^{1,2}$ S. Mombetsu, ${ }^{1,3}$ P. T. Cong, ${ }^{1}$ T. Stöter,,${ }^{1,2}$ E. L. Green $\odot,{ }^{1, *}$ C. Salazar Mejia, ${ }^{1}$ J. Wosnitza, ${ }^{1,2}$ M. Ruminy, \\ T. Fennell $\odot,{ }^{4}$ A. A. Zvyagin,${ }^{5,6,7}$ S. Zherlitsyn $\odot,{ }^{1, \dagger}$ and M. Kenzelmann $\oplus^{4,+}$ \\ ${ }^{1}$ Hochfeld-Magnetlabor Dresden (HLD-EMFL) and Würzburg-Dresden Cluster of Excellence ct.qmat, Helmholtz-Zentrum \\ Dresden-Rossendorf, 01328 Dresden, Germany \\ ${ }^{2}$ Institut für Festkörper- und Materialphysik, Technische Universität Dresden, 01062 Dresden, Germany \\ ${ }^{3}$ Department of Physics, Hokkaido University, Sapporo 060-0810, Japan \\ ${ }^{4}$ Laboratory for Neutron Scattering and Imaging, Paul Scherrer Institut, Forschungssträ̈e 111, 5232 Villigen PSI, Switzerland \\ ${ }^{5}$ Max-Planck-Institut für Physik komplexer Systeme, Nöthnitzer Strasse 38, 01187 Dresden, Germany \\ ${ }^{6}$ B.I. Verkin Institute for Low Temperature Physics and Engineering, National Academy of Sciences of Ukraine, \\ Nauky Avenue 47, Kharkiv 61103, Ukraine \\ ${ }^{7}$ Applied Chemistry Department, V.N. Karazin Kharkiv National University, Svobody sq. 4, Kharkiv 61022, Ukraine
}

(Received 20 January 2020; revised 17 July 2020; accepted 20 July 2020; published 6 August 2020)

\begin{abstract}
Numerous materials feature unexplained phases with invisible or hidden order of electronic origin. A particularly mysterious case is that of $\mathrm{Tb}_{2} \mathrm{Ti}_{2} \mathrm{O}_{7}$, which avoids magnetic order to the lowest temperatures, but nevertheless has an unexplained second-order phase transition near $T=0.5 \mathrm{~K}$. Our ultrasound measurements of $\mathrm{Tb}_{2} \mathrm{Ti}_{2} \mathrm{O}_{7}$ provide direct evidence of a huge softening followed by strong hardening of the structural lattice below $T=0.5 \mathrm{~K}$. In the absence of magnetic order at this temperature, our results provide conclusive evidence for the proposed quadrupolar order and emphasize the importance of higher-order multipolar interactions in rare-earth frustrated magnets.
\end{abstract}

DOI: 10.1103/PhysRevB.102.060403

Many investigations of models in which the interactions between magnetic dipoles are frustrated by lattice geometries including triangular, kagome, or pyrochlore arrangements have been made, outlining the generalities of such systems [1]. This motivated detailed studies of specific consequences such as spin ice and quantum spin ice (with associated emergent fractional quasiparticles) [2-6], order by disorder [7], emergent multipolar degrees of freedom composed of dipoles [8,9], or magnetic moment fragmentation [10,11]. Perhaps because of the wider range of interaction types among higher singleion multipoles [12], along with the expectation that such interactions are too complex to have degenerate frustrated configurations, general investigations of higher multipoles on frustrated lattices are not widespread. However, one of the most diverse families of compounds with pyrochlore lattices are the rare-earth pyrochlores $R_{2} B_{2} \mathrm{O}_{7}$ ( $R$ is a trivalent rareearth ion and $B$ is a tetravalent ion that may be diamagnetic or magnetic; both ions individually form pyrochlore lattices) [13], and it is in the nature of rare-earth ions to carry higher multipole moments. Moreover, it has recently been shown that interactions between the higher multipoles may be of importance in controlling the behavior of frustrated rare-earth systems $[14,15]$.

*Present address: National High Magnetic Field Laboratory, Florida State University, Tallahassee, Florida 32310, USA.

†Corresponding author: s.zherlitsyn@hzdr.de

ॠCorresponding author: michel.kenzelmann@psi.ch
The point symmetry of the rare-earth site in the pyrochlore structure is $D_{3 d}$, which means that the crystal field breaks the degeneracy of the free-ion multiplets down to doublets for Kramers ions, or a mixture of doublets and singlets for non-Kramers ions [16]. In addition to a magnetic dipole moment, a Kramers doublet can carry another magnetic multipole moment, a specific example being the dipolar-octupolar doublets that may provide a route to a type of quantum spin ice $[15,17,18]$. Non-Kramers doublets may have magnetic dipole moments, and are often associated with quadrupolar degrees of freedom. A particular mystery in the field of pyrochlore magnetism is afforded by $\mathrm{Tb}_{2} \mathrm{Ti}_{2} \mathrm{O}_{7}$ $[19,20]$, in which, depending on the exact stoichiometry of the sample [21], a significant specific-heat anomaly may occur at $T \approx 0.5 \mathrm{~K}$, but apparently in no sample does ordering of the full magnetic dipole moment of the non-Kramers doublet occur at any experimentally accessible temperature [22-24]. The specific-heat anomaly has been interpreted as an ordering of quadrupole moments carried by the single-ion ground-state doublet, supported by a theory in which this ground-state doublet is assumed to be thermally isolated [25]. This theory reproduces the specific-heat and recent inelastic neutron-scattering experiments quite well, but cannot explain all aspects of $\mathrm{Tb}_{2} \mathrm{Ti}_{2} \mathrm{O}_{7}$ [24]. Furthermore, there is no direct evidence of the nature of the phase transition, which currently could be described as a hidden order: There is a specific-heat anomaly and redistribution of spectral weight in the excitation spectrum, but no measurable order parameter. Strong coupling between various crystal-field and phonon 
excitations has been evidenced by terahertz spectroscopy [26] and inelastic neutron scattering [27-29], implying the importance of quadrupolar degrees of freedom.

In this work, we use specific-heat, magnetic-susceptibility, and ultrasound measurements to show that a phase transition occurs in $\mathrm{Tb}_{2} \mathrm{Ti}_{2} \mathrm{O}_{7}$, which has a specific-heat and elastic anomaly, but no magnetic anomaly. Because the heatcapacity and ultrasound techniques are sensitive to the ordering of both dipolar and quadrupolar degrees of freedom [30], but the magnetic susceptibility is only sensitive to the ordering of the dipoles, we show directly that the natural explanation of the transition is the ordering of quadrupoles.

Both single crystals, used in this work, have already been described in Ref. [31], where they were named EP2 and EP3 and other pieces of them were found to have slightly different compositions and contrasting specific-heat behavior but identical excitation spectra. The specific heat of the full volume of each sample piece was measured using the heatpulse method in a dilution refrigerator. The (ac) magnetic susceptibility of the same pieces was measured at $16 \mathrm{~Hz}$ using a noncompensated coil connected to an LR700 ac resistance bridge, a physical properties measurement system was used to precool the samples to $2 \mathrm{~K}$, and further cooling was obtained by adiabatic demagnetization of a paramagnetic salt at zero magnetic field. Using a phase-sensitive detection technique [30], we studied the longitudinal $c_{11}(\mathbf{k}\|\mathbf{u}\|[001])$, transverse $c_{44}(\mathbf{k}\|[001], \mathbf{u}\|[100])$, and $\left(c_{11}-c_{12}\right) / 2(\mathbf{k}\|[110], \mathbf{u}\|[1 \overline{1} 0])$ acoustic modes, where $\mathbf{k}$ and $\mathbf{u}$ are the wave vector and polarization, respectively. Sample lengths were $1.775 \mathrm{~mm}$ (EP2) and $2.34 \mathrm{~mm}$ (EP3) for the ultrasound propagation. The elastic modulus, $c_{i j}$, is related to the sound velocity, $v$, by $c_{i j}=$ $\rho v^{2}$, where $\rho$ is the mass density. Resonance $\mathrm{LiNbO}_{3}$ and wide-band polyvinylidene-fluoride-film transducers glued to the sample were employed for sound generation and detection. Before each field sweep, we demagnetized the magnet at a sample temperature of $\approx 2.5 \mathrm{~K}$ and then cooled the sample to the desired temperature (zero-field-cooled condition). For temperature sweeps in applied magnetic field, the field was applied at the base temperature, then the temperature was swept up to $0.8 \mathrm{~K}$ and down to the base temperature at constant magnetic field.

In Fig. 1(a) we show the magnetic susceptibility $\left(\chi^{\prime}\right)$ measured between 0.2 and $10 \mathrm{~K}$ using the full volume of each sample. The susceptibilities of the two samples bifurcate at $T \approx 1 \mathrm{~K}$, but, as in other magnetic susceptibility measurements of $\mathrm{Tb}_{2} \mathrm{Ti}_{2} \mathrm{O}_{7}$, neither shows any strong anomaly that may be associated with conventional magnetic order in the temperature range of these measurements $[21,32,33]$, though there are clear inflections at $T \approx 0.56 \mathrm{~K}$ for EP2 and at $T \approx$ $0.72 \mathrm{~K}$ for EP3. These inflections are less pronounced in some other samples, but a similar one appears at $T \approx 0.6 \mathrm{~K}$ in a sample of $\mathrm{Tb}_{2.005} \mathrm{Ti}_{1.995} \mathrm{O}_{6.9975}$, the sample with the largest and sharpest specific-heat anomaly observed in $\mathrm{Tb}_{2} \mathrm{Ti}_{2} \mathrm{O}_{7}$ [21]. A splitting of the field-cooled/zero-field-cooled susceptibilities is often observed in samples of $\mathrm{Tb}_{2} \mathrm{Ti}_{2} \mathrm{O}_{7}$, but this is typically at $T \sim 0.15 \mathrm{~K}$, just below the lowest temperatures accessed in these measurements. In Fig. 1(b), we show the temperature dependence of the specific heat, again measured using the full volume of both samples. Initial attempts using a small piece cut from the surface of EP2 were inconclusive (having a broad
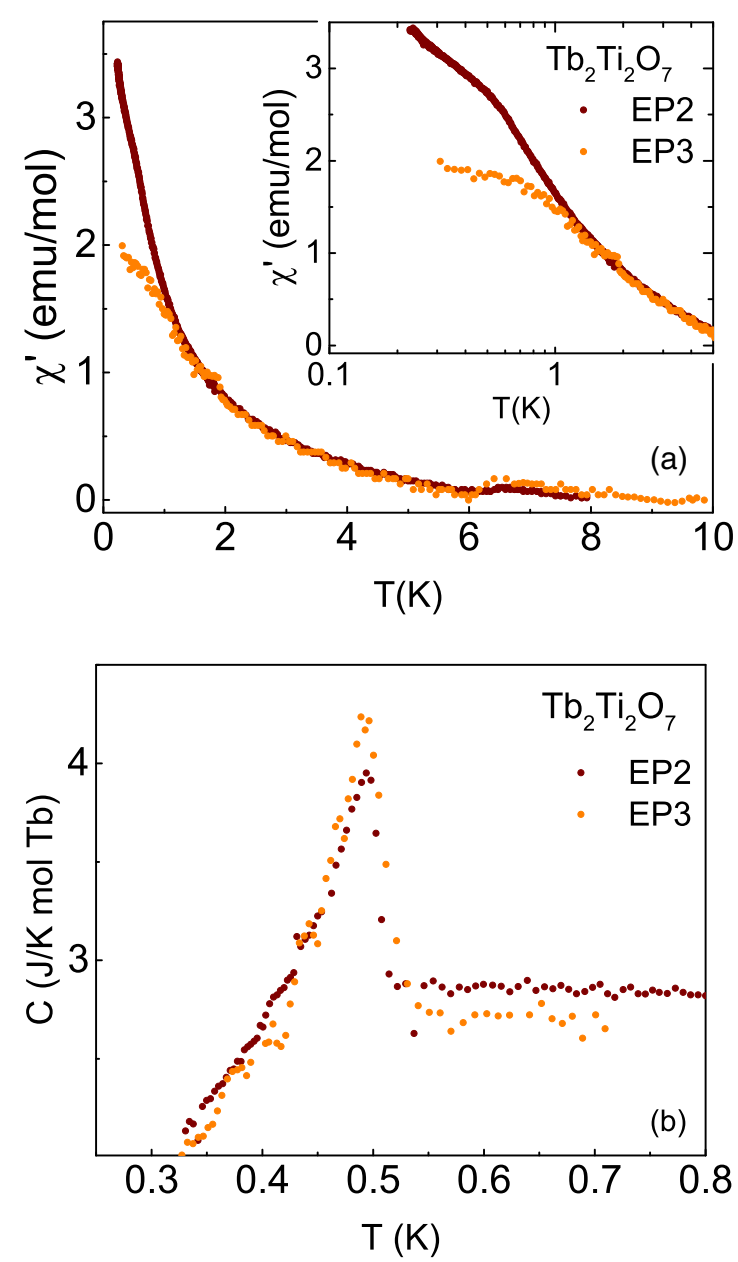

FIG. 1. Temperature dependence of the (a) ac susceptibility and (b) specific heat of the full volume of the EP2 and EP3 samples used for ultrasound experiments. The ac-susceptibility data were obtained in field-cooled condition after adiabatic demagnetization of a paramagnetic salt from $1.8 \mathrm{~K}$ at $5 \mathrm{~T}$. The inset in (a) shows the low-temperature susceptibility in an enlarged and semilogarithmic scale.

and weak feature at $T \approx 0.45 \mathrm{~K}$ ), but a measurement of the full volume of each sample shows that their heat capacities are almost identical [34]. We clearly see a sharp peak in the specific heat indicating a second-order phase transition. Given the absence of a magnetic susceptibility anomaly, this cannot be a magnetic phase transition.

Figures 2(a)-2(c) show the temperature dependence of the relative change of the elastic moduli $c_{11}, c_{44}$, and $\left(c_{11}-\right.$ $\left.c_{12}\right) / 2$ measured at different magnetic fields between 0 and $0.15 \mathrm{~T}$ applied along the [110] direction in samples EP2 [Figs. 2(a) and 2(b)] and EP3 [Fig. 2(c)]. On cooling in zero field, a large [about $64 \%$ for $\left(c_{11}-c_{12}\right) / 2$ and $23 \%$ for $c_{11}$ ] softening of the elastic moduli that starts below $T \approx 100 \mathrm{~K}$ (see also Fig. 3 and Ref. [35]), reaches a pronounced minimum at $0.5 \mathrm{~K}$. This anomaly moves to higher temperatures with increasing magnetic field [36]. An additional anomaly appears in all elastic moduli at about $0.15 \mathrm{~K}$, only when the temperature is increasing. The position of this anomaly also shifts slightly in moderate magnetic fields of 0.075 and $0.15 \mathrm{~T}$, 


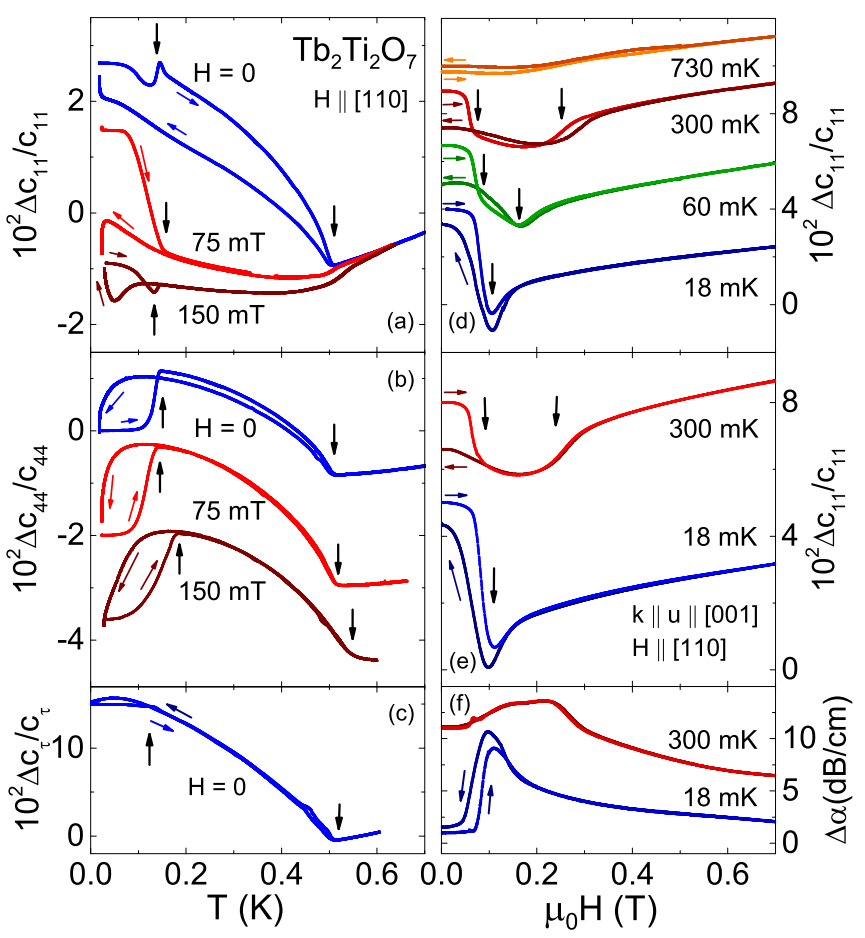

FIG. 2. Temperature dependence of the relative elastic moduli, (a) $\Delta c_{11} / c_{11}$, (b) $\Delta c_{44} / c_{44}$, and (c) $\Delta c_{\tau} / c_{\tau}=\Delta\left(c_{11}-c_{12}\right) /\left(c_{11}-\right.$ $c_{12}$ ) measured at various magnetic fields in $\mathrm{Tb}_{2} \mathrm{Ti}_{2} \mathrm{O}_{7}$ for sample (a), (b) EP2 and (c) EP3. Up and down temperature sweeps are shown. Field dependence of the elastic modulus, $\Delta c_{11} / c_{11}$, for (d) sample EP3, (e) sample EP2, and (f) the sound attenuation, $\Delta \alpha$ in sample EP2. The magnetic field is applied along the [110] direction. Vertical arrows indicate the anomalies in the elastic moduli. The curves for different (a)-(c) magnetic fields and (d)-(f) temperatures are arbitrarily shifted along the $y$ axis for clarity. The ultrasound frequency used was (a), (d) $47 \mathrm{MHz}$, (b) $30 \mathrm{MHz}$, (c) $32 \mathrm{MHz}$, and (e), (f) $41.4 \mathrm{MHz}$.

while the large hysteresis below the $0.5 \mathrm{~K}$ anomaly in $c_{11}$ is suppressed and appears only below $0.15 \mathrm{~K}$ [e.g., Figs. 2(a) and 2(b)].

Our first important observation is the coincidence of the elastic anomaly at $T \approx 0.5 \mathrm{~K}$ with the peak in the heat capacity and the absence of any pronounced anomaly in the magnetic susceptibility for both our samples EP2 and EP3. Although similar combinations of observations using the latter techniques have been recorded for other samples of $\mathrm{Tb}_{2} \mathrm{Ti}_{2} \mathrm{O}_{7}[21,32,37]$, the nature of the phase transition has not been directly identified. The appearance of an elastic and heat-capacity anomaly together with no magnetic anomaly, implies that this phase transition involves degrees of freedom that are strongly coupled to lattice fluctuations and is not a magnetic-ordering transition. Moreover, the magnetic dipole degrees of freedom are essentially unaffected by the transition since the susceptibility continues to evolve smoothly across the transition. These characteristics are commonly associated with an ordering of quadrupoles, which has previously been proposed to occur in $\mathrm{Tb}_{2} \mathrm{Ti}_{2} \mathrm{O}_{7}$ [25]. While various developments of the low-energy part $(E<1 \mathrm{meV})$ of the excitation spectrum are consistent with a theory of such quadrupolar order, the elastic anomaly is a direct experimental signature, and this interpretation is supported by our theoretical consid- erations (see the Supplemental Material [36]; also, references [38-53] therein). The transition apparently represents the end point of the elastic softening that occurs progressively in $\mathrm{Tb}_{2} \mathrm{Ti}_{2} \mathrm{O}_{7}$ below $100 \mathrm{~K}$ (as also shown by previous ultrasound and Young's modulus measurements with a lower limit of $1.8 \mathrm{~K}[35,54,55])$. Due to a lack of lower-temperature data, Refs. $[35,54,55]$ did not provide a proof of the quadrupolar ordering in $\mathrm{Tb}_{2} \mathrm{Ti}_{2} \mathrm{O}_{7}$.

Figures 2(d)-2(f) show the field dependence of $c_{11}$ [(d) for sample EP3 and (e) for sample EP2] together with the attenuation [Fig. 2(f)] of this acoustic mode. At the lowest temperature of $0.018 \mathrm{~K}, c_{11}$ exhibits a pronounced minimum at about $0.1 \mathrm{~T}$ which broadens significantly with temperature increase. The minimum in the elastic modulus is accompanied by a maximum in the sound attenuation. Both elastic properties are characterized by a hysteresis below about $0.1 \mathrm{~T}$. Beyond $0.3 \mathrm{~T}$, up to $0.7 \mathrm{~T}$, only smooth changes in $c_{11}$ and $\Delta \alpha$ have been observed. Both samples exhibit very similar anomalies as a function of applied magnetic field. Again, below $0.5 \mathrm{~K}$ the largest change $(\approx 15 \%)$ is observed for the $\left(c_{11}-c_{12}\right) / 2$ elastic constant [36].

In the inset of Fig. 3, we summarize our observations in the $H-T$ plane for both samples studied. Phase boundaries are tentatively drawn (dashed lines) through the positions of the sharp anomalies (symbols) extracted from our ultrasound experiments. In comparison to works with lower-temperature susceptibility measurements [56] or magnetic neutron

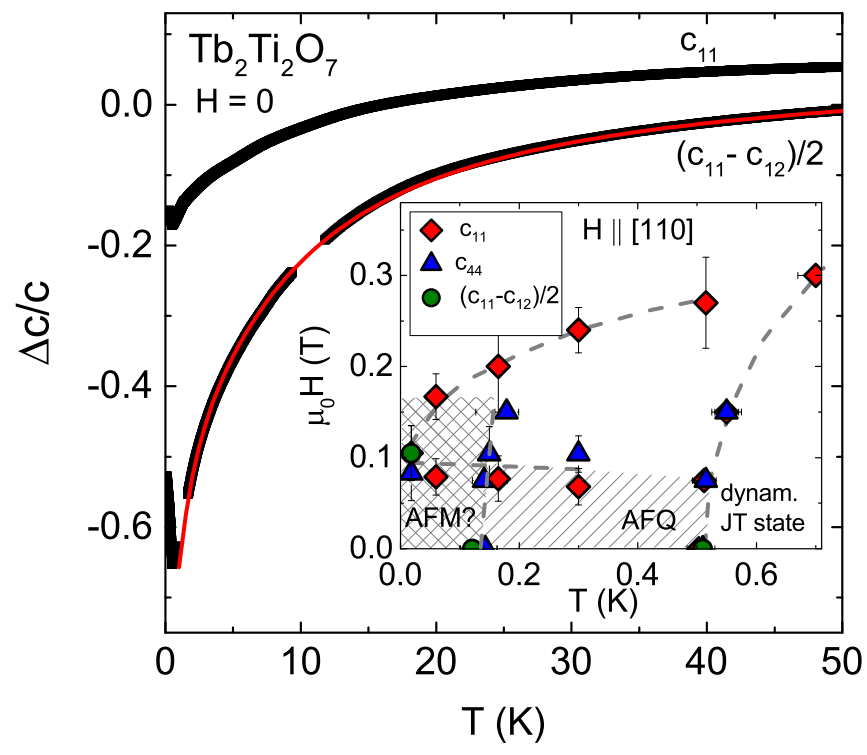

FIG. 3. Temperature dependence of the elastic moduli $\Delta c_{11} / c_{11}$ and $\Delta\left(c_{11}-c_{12}\right) /\left(c_{11}-c_{12}\right)$ (black symbols) obtained in zero magnetic field. The ultrasound frequency was $45 \mathrm{MHz}$ for $\Delta c_{11} / c_{11}$ and $32 \mathrm{MHz}$ for $\Delta\left(c_{11}-c_{12}\right) /\left(c_{11}-c_{12}\right)$. The red line shows the best fit of the data using Eq. (1) with $G=232 \mathrm{~K}$ and $K=-0.055 \mathrm{~K}$ (see text for details). Inset: Positions of the anomalies observed in the acoustic properties of $\mathrm{Tb}_{2} \mathrm{Ti}_{2} \mathrm{O}_{7}$ plotted in the $H-T$ plane for magnetic field applied along the [110] direction. The dashed lines are guides to the eye and separate a dynamical Jahn-Teller regime from the antiferroquadrupolar (AFQ) order, possible antiferromagnetic (AFM) state, and unknown low-temperature field-induced phases beyond $0.1 \mathrm{~T}$. The hatched areas indicate a strong hysteresis in the acoustic properties. 
scattering results [37], the appearance of magnetic anomalies defining a small phase pocket in the region $T<0.15 \mathrm{~K}$ and $0<\mu_{0} H<0.15 \mathrm{~T}$, is consistent. As in Ref. [37], a thermal hysteresis accompanies the formation of this phase, and the boundaries may be frequency dependent. The hightemperature boundary is at a temperature intermediate to susceptibility and neutron-scattering experiments, but since the frequency of the ultrasound experiment is intermediate to the two techniques this is also in agreement with the other studies. This phase is characteriszed by neutron scattering as having short-ranged, frozen spin correlations for the majority of the $\mathrm{Tb}^{3+}$ magnetic moment, with, at most, a very small component of long-range order [22,23,37].

The ultrasound experiments make clear an important aspect of the low-temperature physics of $\mathrm{Tb}_{2} \mathrm{Ti}_{2} \mathrm{O}_{7}$, namely, the nature of the specific-heat anomaly at $T \approx 0.5 \mathrm{~K}$, which appears to be a type of quadrupolar ordering transition. To investigate the nature of the quadrupolar interactions and probable ordering, we use an approach similar to that described in Ref. [30] for the component of the strain related to the elastic modulus $c \equiv\left(c_{11}-c_{12}\right) / 2$. The interaction between the quadrupole components is taken as $K \sum_{i \neq j} O_{\Gamma i} O_{\Gamma j}$, where $K$ is the quadrupole-quadrupole coupling in mean-field approximation, yielding

$$
\frac{\Delta c}{c^{0}}=-\frac{N G^{2}}{c^{0}} \frac{\chi_{q}}{1-K \chi_{q}},
$$

where $c^{0}$ is the nonrenormalized value of the elastic modulus $c, N$ is the number of $\mathrm{Tb}^{3+}$ ions, $\chi_{q}$ is the quadrupole susceptibility of the noninteracting quadrupoles, and $G$ is the quadrupole-strain coupling constant. Here, $\chi_{q}$ contains the contributions from all four $\mathrm{Tb}^{3+}$ sites [36]. Equation (1) implies the softening of the elastic modulus, and that for antiferroquadrupole interactions (i.e., $K<0$ ), $K \chi_{q}$ is negative, analogous to the negative Curie-Weiss temperature of the magnetic susceptibility of magnetic materials with antiferromagnetic interactions. Similar behavior is expected for the renormalization of the elastic modulus $c_{11}$.

In Fig. 3, we show the temperature dependence of the elastic moduli $c_{11}$ and $\left(c_{11}-c_{12}\right) / 2$ over a much larger temperature range, together with the corresponding fit using Eq. (1). The fitted parameters are $G=232 \mathrm{~K}$ and $K=-0.055 \mathrm{~K}$, implying strong coupling between the quadrupoles and lattice, and antiferroquadrupolar interactions, respectively. Although the antiferroquadrupolar interactions suggest that this will be an antiferroquadrupolar order, we cannot deduce the structure from these measurements. However, a $\mathbf{k}=0$ antiferroquadrupolar structure was also suggested in Ref. [25], and supported by the low-temperature evolution of the lowestenergy part of the neutron spectrum. The presence of magnetoelastic modes at considerably higher energies is consistent with the large magnetoelastic coupling parameter [26-29].

Many other examples in which quadrupolar interaction strengths have been quantitatively extracted from the strain susceptibility are metals (where there can be an important contribution to the interactions from the conduction electrons), so it is difficult to find clearly comparable systems $[57,58]$. However, it appears that it is quite possible for the quadrupolar interaction strength to be rather small in com- parison to the ordering temperature, while the magnetoelastic coupling strength may be much larger, i.e., $G \gg K$. In this case, the transition is often referred to as a cooperative JahnTeller effect [59], and the quadrupolar order is entrained, rather than being driven directly by interactions between quadrupoles. However, the possibility of a cooperative JahnTeller effect in $\mathrm{Tb}_{2} \mathrm{Ti}_{2} \mathrm{O}_{7}$ has been debated extensively, and no convincing experimental evidence has yet been advanced [60-66]. Ultimately, the transition is a result of competition between magnetoelastic Jahn-Teller, elastic, magnetic, and quadrupolar interaction energies, as well as entropic contributions to the free energy. The mechanism driving the transition and its energy scale are, therefore, not simple to deduce from the fitted parameters. Careful diffraction and (optical) spectroscopic measurements on samples with wellcontrolled heat capacities would now be of considerable interest.

The phase diagram of Fig. 3 shows that the phase boundary of the antiferroquadrupolar order turns up with field. An upward turning phase boundary, either for quadrupolar or antiferromagnetic phases, is a feature of other quadrupolar systems, including various $R \mathrm{~B}_{2} \mathrm{C}_{2}$ and several other materials, as described in Ref. [67], and references therein. Typically, the field induces order between magnetic multipoles (and may also induce the magnetic multipole itself), and these interactions further stabilize the quadrupolar order. In $\mathrm{Tb}_{2} \mathrm{Ti}_{2} \mathrm{O}_{7}$, it appears that this is only the case for field applied along [110], and does not occur for field applied along [001] or [111]. (The former direction has been studied by ultrasound and not specific heat, and vice versa for the latter directions.) Of course, having multiaxial magnetic anisotropy means that different magnetic structures develop in $\mathrm{Tb}_{2} \mathrm{Ti}_{2} \mathrm{O}_{7}$ when the field is applied along different directions. With the field applied along [110], in the region of the phase diagram of Fig. 3, neutron-scattering experiments show a very rapid growth in Bragg intensity associated with the chainlike ordering of spins on tetrahedron edges parallel to the field (i.e., $\alpha$ chains) $[68,69]$. Apparently this configuration also stabilizes the quadrupolar order, while the "1-in-3-out" order that begins to develop when the field is applied along [111] [65] does not. Detailed comparison of the phase diagrams for magnetic and quadrupolar order could provide a way to understand the competition between magnetic and (so far unconsidered) quadrupolar interactions in $\mathrm{Tb}_{2} \mathrm{Ti}_{2} \mathrm{O}_{7}$, which would be an important step in understanding the parameters of the Hamiltonian of $\mathrm{Tb}_{2} \mathrm{Ti}_{2} \mathrm{O}_{7}$.

In summary, the elastic properties of $\mathrm{Tb}_{2} \mathrm{Ti}_{2} \mathrm{O}_{7}$ show a number of anomalies, both versus temperature and magnetic field. In particular, a pronounced $\lambda$ anomaly in the specific heat and the absence of a clear anomaly in the magnetic susceptibility evidence a nonmagnetic phase transition at $0.5 \mathrm{~K}$ in $\mathrm{Tb}_{2} \mathrm{Ti}_{2} \mathrm{O}_{7}$. The large softening of the acoustic modes below $100 \mathrm{~K}$ and the elastic-constant anomaly at $0.5 \mathrm{~K}$ with a hysteresis below $0.5 \mathrm{~K}$, detected in both studied single crystals, signal an antiferroquadrupolar ordering. Theoretical considerations based on analysis of the crystal electric field of $\mathrm{Tb}_{2} \mathrm{Ti}_{2} \mathrm{O}_{7}$ support our experimental findings [36]. The current interest in non-Kramers doublet systems, particularly based on $\operatorname{Pr}^{3+}$ [70-72], on the pyrochlore lattice means understanding quadrupolar degrees of freedom and interactions with this 
lattice is of some importance. For example, in $\operatorname{Pr}_{2} \mathrm{Zr}_{2} \mathrm{O}_{7}$, it is proposed that the ground state is an antiferroquadrupolar liquid [73], while in $\mathrm{Pr}_{2} \mathrm{Hf}_{2} \mathrm{O}_{7}$ the quadrupolar interactions are relatively less important, resulting in a spin-liquid ground state [74]. $\mathrm{Tb}_{2} \mathrm{Ti}_{2} \mathrm{O}_{7}$ may serve as a useful counterpoint, in which the factors that control quadrupolar order in rare-earth pyrochlores can be investigated.
We thank Roderich Moessner and Tatsuya Yanagisawa for helpful discussions and suggestions. We acknowledge support of the HLD at HZDR, member of the European Magnetic Field Laboratory (EMFL), and the DFG through SFB 1143 and through the Würzburg-Dresden Cluster of Excellence on Complexity and Topology in Quantum Matter-ct.qmat (EXC 2147, Project No. 390858490).
[1] C. Lacroix, P. Mendels, and F. Mila, Introduction to Frustrated Magnetism (Springer, Berlin, 2010).

[2] M. J. Harris, S. T. Bramwell, D. F. McMorrow, T. Zeiske, and K. W. Godfrey, Phys. Rev. Lett. 79, 2554 (1997).

[3] M. Hermele, M. P. A. Fisher, and L. Balents, Phys. Rev. B 69, 064404 (2004).

[4] C. Castelnovo, R. Moessner, and S. L. Sondhi, Nature (London) 451, 42 (2008).

[5] C. Castelnovo, R. Moessner, and S. L. Sondhi, Annu. Rev. Condens. Matter Phys. 3, 35 (2012).

[6] M. J. P. Gingras and P. A. McClarty, Rep. Prog. Phys. 77, 056501 (2014).

[7] L. Savary, K. A. Ross, B. D. Gaulin, J. P. C. Ruff, and L. Balents, Phys. Rev. Lett. 109, 167201 (2012).

[8] M. E. Zhitomirsky, Phys. Rev. B 78, 094423 (2008).

[9] J. A. M. Paddison, H. Jacobsen, O. A. Petrenko, M. T. Fernandez-Diaz, P. P. Deen, and A. L. Goodwin, Science 350, 179 (2015).

[10] M. E. Brooks-Bartlett, S. T. Banks, L. D. C. Jaubert, A. Harman-Clarke, and P. C. W. Holdsworth, Phys. Rev. X 4, 011007 (2014).

[11] S. Petit, E. Lhotel, B. Canals, M. Ciomaga Hatnean, J. Ollivier, H. Mutka, E. Ressouche, A. R. Wildes, M. R. Lees, and G. Balakrishnan, Nat. Phys. 12, 746 (2016).

[12] P. Santini, S. Carretta, G. Amoretti, R. Caciuffo, N. Magnani, and G. H. Lander, Rev. Mod. Phys. 81, 807 (2009).

[13] J. S. Gardner, M. J. P. Gingras, and J. E. Greedan, Rev. Mod. Phys. 82, 53 (2010).

[14] N. Gauthier, A. Fennell, B. Prévost, A. Désilets-Benoit, H. A. Dabkowska, J. Ollivier, J. S. White, W. Schmitt, L.-P. Regnault, F. Bourdarot, A. D. Bianchi, and M. Kenzelmann (unpublished).

[15] R. Sibille, N. Gauthier, E. Lhotel, V. Porée, V. Pomjakushin, R. A. Ewings, T. G. Perring, J. Ollivier, A. Wildes, C. Ritter, T. C. Hansen, D. A. Keen, G. J. Nilsen, L. Keller, S. Petit, and T. Fennell, Nat. Phys. 16, 546 (2020).

[16] U. Walter, J. Phys. Chem. Solids 45, 401 (1984).

[17] Y.-P. Huang, G. Chen, and M. Hermele, Phys. Rev. Lett. 112, 167203 (2014).

[18] Y.-D. Li and G. Chen, Phys. Rev. B 95, 041106(R) (2017).

[19] H. R. Molavian, M. J. P. Gingras, and B. Canals, Phys. Rev. Lett. 98, 157204 (2007).

[20] J. G. Rau and M. J. P. Gingras, Annu. Rev. Condens. Matter Phys. 10, 357 (2018).

[21] T. Taniguchi, H. Kadowaki, H. Takatsu, B. Fak, J. Ollivier, T. Yamazaki, T. J. Sato, H. Yoshizawa, Y. Shimura, T. Sakakibara, T. Hong, K. Goto, L. R. Yaraskavitch, and J. B. Kycia, Phys. Rev. B 87, 060408(R) (2013).

[22] S. Guitteny, I. Mirebeau, P. Dalmas de Réotier, C. V. Colin, P. Bonville, F. Porcher, B. Grenier, C. Decorse, and S. Petit, Phys. Rev. B 92, 144412 (2015).
[23] E. Kermarrec, D. D. Maharaj, J. Gaudet, K. Fritsch, D. Pomaranski, J. B. Kycia, Y. Qiu, J. R. D. Copley, M. M. P. Couchman, A. O. R. Morningstar, H. A. Dabkowska, and B. D. Gaulin, Phys. Rev. B 92, 245114 (2015).

[24] H. Kadowaki, M. Wakita, B. Fåk, J. Ollivier, S. OhiraKawamura, K. Nakajima, and J. W. Lynn, Phys. Rev. B 99, 014406 (2019).

[25] H. Takatsu, S. Onoda, S. Kittaka, A. Kasahara, Y. Kono, T. Sakakibara, Y. Kato, B. Fåk, J. Ollivier, J. W. Lynn, T. Taniguchi, M. Wakita, and H. Kadowaki, Phys. Rev. Lett. 116, 217201 (2016).

[26] E. Constable, R. Ballou, J. Robert, C. Decorse, J.-B. Brubach, P. Roy, E. Lhotel, L. Del-Rey, V. Simonet, S. Petit, and S. de Brion, Phys. Rev. B 95, 020415 (2017).

[27] T. Fennell, M. Kenzelmann, B. Roessli, H. Mutka, J. Ollivier, M. Ruminy, U. Stuhr, O. Zaharko, L. Bovo, A. Cervellino, M. K. Haas, and R. J. Cava, Phys. Rev. Lett. 112, 017203 (2014).

[28] M. Ruminy, E. Pomjakushina, K. Iida, K. Kamazawa, D. T. Adroja, U. Stuhr, and T. Fennell, Phys. Rev. B 94, 024430 (2016).

[29] M. Ruminy, S. Guitteny, J. Robert, L. P. Regnault, M. Boehm, P. Steffens, H. Mutka, J. Ollivier, U. Stuhr, J. S. White, B. Roessli, L. Bovo, C. Decorse, M. K. Haas, R. J. Cava, I. Mirebeau, M. Kenzelmann, S. Petit, and T. Fennell, Phys. Rev. B 99, 224431 (2019).

[30] B. Lüthi, Physical Acoustics in the Solid State (Springer, Berlin, 2005).

[31] M. Ruminy, L. Bovo, E. Pomjakushina, M. K. Haas, U. Stuhr, A. Cervellino, R. J. Cava, M. Kenzelmann, and T. Fennell, Phys. Rev. B 93, 144407 (2016).

[32] N. Hamaguchi, T. Matsushita, N. Wada, Y. Yasui, and M. Sato, Phys. Rev. B 69, 132413 (2004).

[33] E. Lhotel, C. Paulsen, P. D. de Réotier, A. Yaouanc, C. Marin, and S. Vanishri, Phys. Rev. B 86, 020410(R) (2012).

[34] In Ref. [31] some of us found the heat capacity of small pieces of these two samples (different to those used here) to be different, but the excitation spectra (for energy transfers above $4 \mathrm{meV}$ ) measured using the much larger original boules of each to be quantitatively identical. The combination of these observations and the previous ones supports the claim that $\mathrm{Tb}_{2} \mathrm{Ti}_{2} \mathrm{O}_{7}$ crystals can be inhomogeneous [75], but does not invalidate our earlier conclusion that the higher-energy excitations, when measured in the full volume of large $\mathrm{Tb}_{2} \mathrm{Ti}_{2} \mathrm{O}_{7}$ crystals, are generic.

[35] Y. Nakanishi, T. Kumagai, M. Yoshizawa, K. Matsuhira, S. Takagi, and Z. Hiroi, Phys. Rev. B 83, 184434 (2011).

[36] See Supplemental Material at http://link.aps.org/supplemental/ 10.1103/PhysRevB.102.060403 for additional ultrasound results, further details of the crystal electric field analysis, and 
theoretical consideration of sound characteristics in the strainexchange approximation.

[37] K. Fritsch, E. Kermarrec, K. A. Ross, Y. Qiu, J. R. D. Copley, D. Pomaranski, J. B. Kycia, H. A. Dabkowska, and B. D. Gaulin, Phys. Rev. B 90, 014429 (2014).

[38] K. W. H. Stevens, Proc. Phys. Soc. A 65, 209 (1952).

[39] M. J. P. Gingras, B. C. den Hertog, M. Faucher, J. S. Gardner, S. R. Dunsiger, L. J. Chang, B. D. Gaulin, N. P. Raju, and J. E. Greedan, Phys. Rev. B 62, 6496 (2000).

[40] V. V. Klekovkina and B. Z. Malkin, Opt. Spectrosc. 116, 849 (2014).

[41] A. Bertin, Y. Chapuis, P. Dalmas de Réotier, and A. Yaouanc, J. Phys.: Condens. Matter 24, 256003 (2012).

[42] I. V. Aleksandrov, B. V. Lidskii, L. G. Mamsurova, M. G. Neigauz, K. S. Pigal'skii, K. K. Pukhov, N. G. Trusevich, and L. G. Shcherbakova, Sov. Phys. JETP 62, 1287 (1985).

[43] J. Zhang, K. Fritsch, Z. Hao, B. V. Bagheri, M. J. P. Gingras, G. E. Granroth, P. Jirammongkolchai, R. J. Cava, and B. D. Gaulin, Phys. Rev. B 89, 134410 (2014).

[44] I. Mirebeau, P. Bonville, and M. Hennion, Phys. Rev. B 76, 184436 (2007).

[45] A. J. Princep, H. C. Walker, D. T. Adroja, D. Prabhakaran, and A. T. Boothroyd, Phys. Rev. B 91, 224430 (2015).

[46] S. H. Curnoe, Phys. Rev. B 78, 094418 (2007).

[47] P. A. McClarty, S. H. Curnoe, and M. J. P. Gingras, J. Phys.: Conf. Ser. 145, 012032 (2009).

[48] R. Moessner, Phys. Rev. B 57, R5587(R) (1998).

[49] R. Moessner and J. T. Chalker, Phys. Rev. B 58, 12049 (1998).

[50] S. V. Isakov, K. Gregor, R. Moessner, and S. L. Sondhi, Phys. Rev. Lett. 93, 167204 (2004).

[51] A. A. Zvyagin, Fiz. Nizk. Temp. 39, 1159 (2013) [Low Temp. Phys. 39, 901 (2013)].

[52] O. Ofer, A. Keren, and C. Baines, J. Phys.: Condens. Matter 19, 145270 (2007).

[53] P. Bonville, A. Gukasov, I. Mirebeau, and S. Petit, Phys. Rev. B 89, 085115 (2014).

[54] L. G. Mamsurova, K. S. Pigal'skii, K. K. Pukhov, N. G. Trusevich, and L. G. Shcherbakova, Sov. Phys. JETP 67, 550 (1988).

[55] L. G. Mamsurova, K. S. Pigal'skii, and K. K. Pukhov, JETP Lett. 43, 755 (1986).

[56] L. Yin, J. S. Xia, Y. Takano, N. S. Sullivan, Q. J. Li, and X. F. Sun, Phys. Rev. Lett. 110, 137201 (2013).
[57] P. M. Levy, P. Morin, and D. Schmitt, Phys. Rev. Lett. 42, 1417 (1979).

[58] P. Morin and D. Schmitt, Handbook of Ferromagnetic Materials (North-Holland, Amsterdam, 1990), Vol. 5, p. 1.

[59] G. A. Gehring and K. A. Gehring, Rep. Prog. Phys. 38, 1 (1975).

[60] J. P. C. Ruff, B. D. Gaulin, J. P. Castellan, K. C. Rule, J. P. Clancy, J. Rodriguez, and H. A. Dabkowska, Phys. Rev. Lett. 99, 237202 (2007).

[61] K. C. Rule and P. Bonville, J. Phys.: Conf. Ser. 145, 012027 (2009).

[62] P. Bonville, I. Mirebeau, A. Gukasov, S. Petit, and J. Robert, Phys. Rev. B 84, 184409 (2011).

[63] B. D. Gaulin, J. S. Gardner, P. A. McClarty, and M. J. P. Gingras, Phys. Rev. B 84, 140402(R) (2011).

[64] A. Yaouanc, P. Dalmas de Réotier, Y. Chapuis, C. Marin, S. Vanishri, D. Aoki, B. Fåk, L. P. Regnault, C. Buisson, A. Amato, C. Baines, and A. D. Hillier, Phys. Rev. B 84, 184403 (2011).

[65] A. P. Sazonov, A. Gukasov, H. B. Cao, P. Bonville, E. Ressouche, C. Decorse, and I. Mirebeau, Phys. Rev. B 88, 184428 (2013).

[66] M. Ruminy, F. Groitl, T. Keller, and T. Fennell, Phys. Rev. B 94, 174406 (2016).

[67] K. Kaneko, H. Onodera, H. Yamauchi, T. Sakon, M. Motokawa, and Y. Yamaguchi, Phys. Rev. B 68, 012401 (2003).

[68] K. C. Rule, J. P. C. Ruff, B. D. Gaulin, S. R. Dunsiger, J. S. Gardner, J. P. Clancy, M. J. Lewis, H. A. Dabkowska, I. Mirebeau, P. Manuel, Y. Qiu, and J. R. D. Copley, Phys. Rev. Lett. 96, 177201 (2006).

[69] A. P. Sazonov, A. Gukasov, I. Mirebeau, H. Cao, P. Bonville, B. Grenier, and G. Dhalenne, Phys. Rev. B 82, 174406 (2010).

[70] S. Onoda and Y. Tanaka, Phys. Rev. Lett. 105, 047201 (2010).

[71] S. Onoda and Y. Tanaka, Phys. Rev. B 83, 094411 (2011).

[72] S. B. Lee, S. Onoda, and L. Balents, Phys. Rev. B 86, 104412 (2012).

[73] S. Petit, E. Lhotel, S. Guitteny, O. Florea, J. Robert, P. Bonville, I. Mirebeau, J. Ollivier, H. Mutka, E. Ressouche, C. Decorse, M. Ciomaga Hatnean, and G. Balakrishnan, Phys. Rev. B 94, 165153 (2016).

[74] R. Sibille, N. Gauthier, H. Yan, M. C. Hatnean, J. Ollivier, B. Winn, U. Filges, G. Balakrishnan, M. Kenzelmann, N. Shannon, and T. Fennell, Nat. Phys. 14, 711 (2018).

[75] M. Wakita, T. Taniguchi, H. Edamoto, H. Takatsu, and H. Kadowaki, J. Phys.: Conf. Ser. 683, 012023 (2016). 